\title{
The effect of number of trials per day during sequential alternation of unilateral amygdaloid stimulation
}

\author{
JOHN GAITO \\ York University, 4700 Keele Street, Downsview, MSJ 1PS, Ontario, Canada
}

\begin{abstract}
Three groups of rats were subjected to a sequence of stimulations alternating from one amygdala to the contralateral one. Each phase of stimulation was for six convulsions prior to stimulation of the opposite side. Rats in Group 1 were stimulated six times per day; Group 2 rats had three trials each day; and one stimulation each day was provided for Group 3 rats. The oscillation tendency (high values for one side, low values for other side) was prominent with all groups but seemed most prevalent with Group 3 rats.
\end{abstract}

Previous research indicated an "oscillation effect" when a sequence of alternating unilateral stimulations of the amygdala were utilized (Gaito, 1975). This oscillation consisted usually of low values for the amygdala first stimulated (primary site) and high values for the contralateral amygdala (secondary site). The effect was most prominent in latency data. Bilateral stimulation produced little effect on this oscillation tendency (Gaito, 1976). In this experiment, the effects of number of trials per day on the oscillation tendency was investigated.

\section{METHOD}

Twenty-five male Wistar rats (approximately 135 days of age) were implanted bilaterally with bipolar electrodes in the amygdala. The brain coordinates for electrode implantation were the same as in previous experiments: $.5 \mathrm{~mm}$ posterior to bregma, $4.5 \mathrm{~mm}$ from midline, $8.5 \mathrm{~mm}$ from skull.

Seven or more days after surgery, brain stimulation began. Each rat in one group of eight (Group 1) was stimulated for $30 \mathrm{sec}$ with a $60-\mathrm{Hz}$ wave of 100 microA (peak to peak) using a Lafayette stimulator until six convulsions resulted. There were six trials per day, with $1 \mathrm{~h}$ between trials. On the day after the sixth convulsion, stimulation began at the secondary site and continued until six convulsions occurred. Then stimulation again at the primary site was instituted, etc. This sequential alternation of unilateral stimulation was continued for 10 phases.

In Group 2, eight rats were treated in a similar fashion except that only three trials per day were used. In Group 3, nine rats had a single trial each day.

Some rats did not reach a clonic convulsion (CC) within 30 trials. Then the intensity was slowly increased over trials, to a maximum of 560 microA with a few rats, until a CC occurred.

\section{RESULTS}

The results for the three groups are shown in Table 1 for the two dependent variables: latency (number of seconds between onset of stimulation and onset of CC), criterion data (number of trials to six CC). As in previous work (Gaito, 1975, 1976), an oscillation effect appeared which was stronger in latency than in criterion data. In the three groups, the number of oscillators in the latency data were four,
Table 1

Number of Rats Showing Oscillation (0) or No Oscillation (NO) for the Three Groups

\begin{tabular}{lccccccc}
\hline & Group 1(6X)* & \multicolumn{2}{c}{ Group 2 (3X)† } & \multicolumn{3}{c}{ Group $3(1 \mathrm{X})$} \\
& O & NO & O & NO & O & NO \\
\hline Latency & 4 & 2 & 5 & 2 & 9 & 0 \\
Criterion & 3 & 3 & 3 & 4 & 6 & 3 \\
\hline
\end{tabular}

*Two rats had to be discarded because CCs were not attained on both sides.

tOne rat lost electrode.

five, and nine, respectively. Thus 18 rats oscillated out of the 22 rats which completed the 10 phases. Only 12 rats oscillated in criterion data.

Two rats in Group 1 were discarded because no CC had resulted by 60 trials. A visual examination of each brain using a magnifying lens indicated that the electrode was outside the amygdala in one electrode in both cases. In one case the electrode was in the internal capsule; in the other, the electrode was in the cortex next to the rhinal fissure. One rat in Group 2 was lost when the electrode came loose.

In latency data, there were 11 primary oscillators (low values in primary site, high values in secondary site)-five secondary oscillators (high values in primary site, low values in secondary site) and two with both primary and secondary oscillation for a series of at least four phases each. For criterion data there were seven primary oscillators, four secondary oscillators, and one mixed oscillator.

No statistical analyses were attempted to compare the three groups relative to the number of oscillators. However, there is the suggestion that oscillation is more apt to result with one stimulation per day. Such results may be consistent with those of Goddard, McIntyre, and Leech (1969) who reported that one trial per day produced more efficient kindling.

Typical oscillation data are provided in Table 2. Rat 4 did not begin to show oscillation in latency until 
Table 2

Typical Oscillation for One Rat in Each Group

\begin{tabular}{|c|c|c|c|c|c|c|c|c|c|c|}
\hline & \multicolumn{10}{|c|}{ Phases } \\
\hline & 1 & 2 & 3 & 4 & 5 & 6 & 7 & 8 & 9 & 10 \\
\hline & \multicolumn{10}{|c|}{ Group 1 (Rat 4) } \\
\hline \multirow{3}{*}{ Criterion } & 13.5 & 5.7 & 8.0 & 12.3 & 5.5 & 18.0 & 8.3 & 23.2 & 4.8 & 23.2 \\
\hline & 36 & 51 & 6 & 6 & 6 & 9 & 6 & 9 & 6 & 10 \\
\hline & \multicolumn{10}{|c|}{ Group 2 (Rat 11) } \\
\hline \multirow{3}{*}{$\begin{array}{l}\text { Latency } \\
\text { Criterion }\end{array}$} & 3.0 & 11.5 & 3.8 & 10.7 & 5.3 & 10.2 & 4.5 & 18.5 & 5.3 & 14.2 \\
\hline & 61 & 14 & 6 & 6 & 7 & 6 & 7 & 7 & 6 & 8 \\
\hline & \multicolumn{10}{|c|}{ Group 3 (Rat 18) } \\
\hline Latency & 9.2 & 3.3 & 12.5 & 2.8 & 15.3 & 3.0 & 10.7 & 4.5 & 6.3 & 2.3 \\
\hline Criterion & 17 & 15 & 10 & 6 & 8 & 6 & 6 & 6 & 6 & 6 \\
\hline
\end{tabular}

Phase 3, after a single stimulation of each site. The two other rats showed oscillation throughout the 10 phases in the latency measure.

\section{DISCUSSION}

These results are consistent with the previous ones (Gaito, 1975, 1976) which demonstrated the oscillation tendency and indicated that bilateral stimulation had little effect on this tendency. Number of trials per day (one, three, six) also appears not to disrupt the oscillation tendency, although one trial per day may provide the most favorable occasion for it to occur. The previous research used three trials each day.

These results also indicate the authenticity of the oscillation effect. This tendency appears to be a prominent feature of unilateral sequential alternations and is not due to chance aspects or to poorly placed electrodes in one of the two sites (Gaito, 1975. 1976).

As in the previous research, there was again the suggestion that an active inhibitory process is generated during unilateral stimulation which affects that site or the contralateral site during subsequent stimulations. For example, Rat 24 of Group 3 reared on hind paws quickly and began to $\mathrm{CC}$ within a few seconds when stimulating the primary site. When stimulating the secondary site. this rat reared as quickly as when stimulating the primary site; however. the CC did not result until after at least $6 \mathrm{sec}$ had elapsed. In some cases, the front paws would move slightly as if the $\mathrm{CC}$ were beginning. However, this movement would terminate within 1 or $2 \mathrm{sec}$. The rat appeared to be actively inhibited during this state of suspension prior to convulsing.

\section{REFERENCES}

GAITO, J. An oscillation effect during sequential alternations of unilateral amygdaloid stimulations within the kindling paradigm. Submitted for publication, 1975.

GarTo, J. The effect of bilateral stimulation during sequential alternation of unilateral amygdaloid stimulation. Bulletin of the Psychonomic Society, 1976, in press.

Goddard, G. V.. McIntyre, D. C.. \& Leech, C. K. A permanent change in brain function resulting from daily electrical stimulation. Experimental Neurology, 1969, 25. 295-330.

(Received for publication January 9, 1976) 